\title{
Neglected tuberculous trochanteric bursitis in an adolescent girl: A case report and literature review
}

\author{
Josip Vlaic, MD, $\mathrm{PhD}^{1}$ (D), Ivan Pavic, MD, $\mathrm{PhD}^{2,3}{ }^{\mathbb{D}}$, Ana Tripalo Batos, $\mathrm{MD}, \mathrm{PhD}^{4}$, \\ Ljiljana Zmak, MD, PhD 5,6 , Bozo Kruslin, MD, PhD ${ }^{7,8}$ (D) \\ 'Division of Paediatric Orthopaedic Surgery, Children's Hospital Zagreb, Zagreb, Croatia \\ ²Department of Pulmonology, Allergology and Immunology, Children's Hospital Zagreb, Zagreb, Croatia \\ ${ }^{3}$ Department of Paediatrics, School of Medicine, University of Split, Split, Croatia \\ ${ }^{4}$ Department of Paediatric Radiology, Children's Hospital Zagreb, Zagreb, Croatia \\ ${ }^{5}$ National Reference Laboratory for Tuberculosis, Croatian National Institute of Public Health, Zagreb, Croatia \\ ${ }^{6}$ Department of Medical Microbiology and Parasitology, School of Medicine, University of Zagreb, Zagreb, Croatia \\ ${ }^{7}$ Department of Pathology, Sestre Milosrdnice University Hospital Centre, Zagreb, Croatia \\ ${ }^{8}$ Department of Pathology, School of Medicine, University of Zagreb, Zagreb, Croatia
}

\begin{abstract}
Extrapulmonary tuberculosis (TB) occurred in $15 \%$ of all TB cases globally in 2018, while musculoskeletal TB accounts for approximately $10 \%$ of all extrapulmonary TB cases in developed

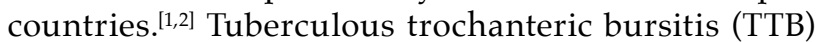
is uncommon and accounts for less than $2 \%$ of musculoskeletal TB. ${ }^{[3]}$ However, in the first half of the $20^{\text {th }}$ century, TTB was not as uncommon as today. ${ }^{[4,5]}$ Longstanding medical literature until 1950 described only 105 cases of TTB, not distinguishing between isolated infection of the bursa, trochanter, or combine infection of both the bursa and trochanter. ${ }^{[6]}$
\end{abstract}

Received: January 29, 2021

Accepted: March 21, 2021

Published online: June 11, 2021

Correspondence: Josip Vlaic, MD, PhD. Division of Paediatric

Orthopaedic Surgery, Children's Hospital Zagreb, Ulica

Vjekoslava Klaica 16; 10000 Zagreb, Croatia.

E-mail: jvlaic@yahoo.co.uk

Doi: $10.52312 /$ jdrs.2021.17

Citation: Vlaic J, Pavic I, Batos AT, Zmak L, Kruslin B. Neglected tuberculous trochanteric bursitis in an adolescent girl: A case report and literature review. Jt Dis Relat Surg 2021;32(2):536-541.

(C2021 All right reserved by the Turkish Joint Diseases Foundation

This is an open access article under the terms of the Creative Commons Attribution-NonCommercial License, which permits use, distribution and reproduction in any medium, provided the original work is properly cited and is not used for commercial purposes (http://creativecommons.org/licenses/by-nc/4.0/).

\section{ABSTRACT}

Tuberculous trochanteric bursitis (TTB) is an extremely rare form of extrapulmonary tuberculosis. Due to a low clinical suspicion and poor collaboration among medical professionals, the diagnosis of TTB can be often delayed. In this report, we describe a case of neglected TTB in an adolescent girl that initially presented with right thigh swelling and fluctuance. The patient underwent repeated unsuccessful surgical treatment; however, dull pain and periodic wound drainage remained for eight years. Complete excision of fistula and trochanteric bursa and one year of oral antituberculous drug therapy led to complete recovery. This case report highlights tuberculosis as a diagnostic challenge, when rare localizations are affected. In addition, this report addresses several diagnostic pitfalls and reviews the literature regarding TTB in adolescent patients. Orthopedic surgeons need to consider TTB, when swelling, fluctuance or repeated wound drainage are present on the thigh.

Keywords: Adolescent, anti-tuberculosis drugs, surgery, tuberculous trochanteric bursitis.

Recently, most of the published data on this subject are case series and case reports with only two adolescent patients reported. ${ }^{[3,7,8]}$

Mainly due to a low clinical suspicion, lack of symptoms, and poor collaboration among medical professionals, the diagnosis of TTB can be delayed. Therefore, it is not surprising that TTB may be easily overlooked and neglected, delaying treatment and affect the patient's quality of life.

In this report, we present a case of TTB in an immunocompetent adolescent who initially presented 
with recurrent thigh swelling. After several unsuccessful treatment regimens over eight years, she remained symptomatic, developing prolonged wound drainage of a cutaneous fistula. This report addresses several diagnostic pitfalls in ТTВ confirmation and reviews the literature regarding epidemiology of TTB in adolescent patients.

\section{CASE REPORT}

\section{Patient's history}

A 14-year-old girl presented to the tertiary referral Children's Hospital orthopedic outpatient clinic due to dull pain and periodic wound drainage on the lateral side of her right thigh. Her mother noted two previous surgeries on that area performed at a county hospital. The first one, eight years ago, was performed due to right thigh swelling and fluctuance. These symptoms were initially suspected to be caused by pyomyositis of the vastus lateralis. A small longitudinal incision on the lateral side of the thigh was done and a purulent cyst was evacuated. Samples were sent for microbiological, cytological, and pathological examination. The laboratory reports were not evaluated properly by the treating surgeon, and the girl was discharged from the hospital with recommendations for oral antibiotics to treat pyomyositis. A month after the operation, purulent wound drainage appeared. The child was continued to treated with oral antibiotics and underwent frequent dressing changes, until complete wound closure was achieved. However, 10 months later, swelling, pain and purulent drainage reappeared at the place of the initial incision. The patient underwent repeated surgical incision and evacuation of what was considered to be a recurrent subfascial abscess. After surgery, the patient was instructed to continue with antibiotics and to dressing changes while waiting for wound closure. Unfortunately, her wound was not completely closed. For the next seven years, she suffered from monthly purulent drainage accompanied by moderate swelling and dull thigh pain that was most intense a few days before the appearance of drainage. During that period, there were no complaints about fever or malaise.

\section{Physical examination and initial considerations}

Seven years after the second surgery, physical examination performed at our institution showed a chronic wound on the lateral right thigh with mild drainage and local erythema. Palpation was slightly painful; however, there was full, painless range of motion of the hip and knee. A swab was taken for microbiological examination and an initial laboratory work-up was done. Microbiology results became positive for Pseudomonas stutzeri, while the laboratory findings were within the normal range. Initial radiographs of the femur were deemed normal. A thigh ultrasound scan revealed a sharply bordered hypoechoic collection between muscles and subcutaneous fat tissue that measured $3 \times 1 \mathrm{~cm}$ in size, which was considered to be an abscess. Oral ciprofloxacin 1,000 $\mathrm{mg}$ was prescribed for 10 days; however, there was no change in her exam. Therefore, due to clinical presentation and according to previous diagnostic work-up findings, recurrent abscess was suspected. The patient was scheduled for surgical excision followed by continuous intravenous antibiotic therapy. However, while waiting for surgery, she developed intense pain in the right hip.

\section{Radiological findings}

Hip radiographs revealed a lytic lesion within the greater trochanter with partial reactive sclerosis (Figure 1). Unfortunately, due to the initial symptoms, as well as the site of the previous surgeries and ultrasound findings, surgery was performed without additional imaging studies.

\section{Surgical technique}

Surgery started with scar tissue excision around the previous wound. However, due to the

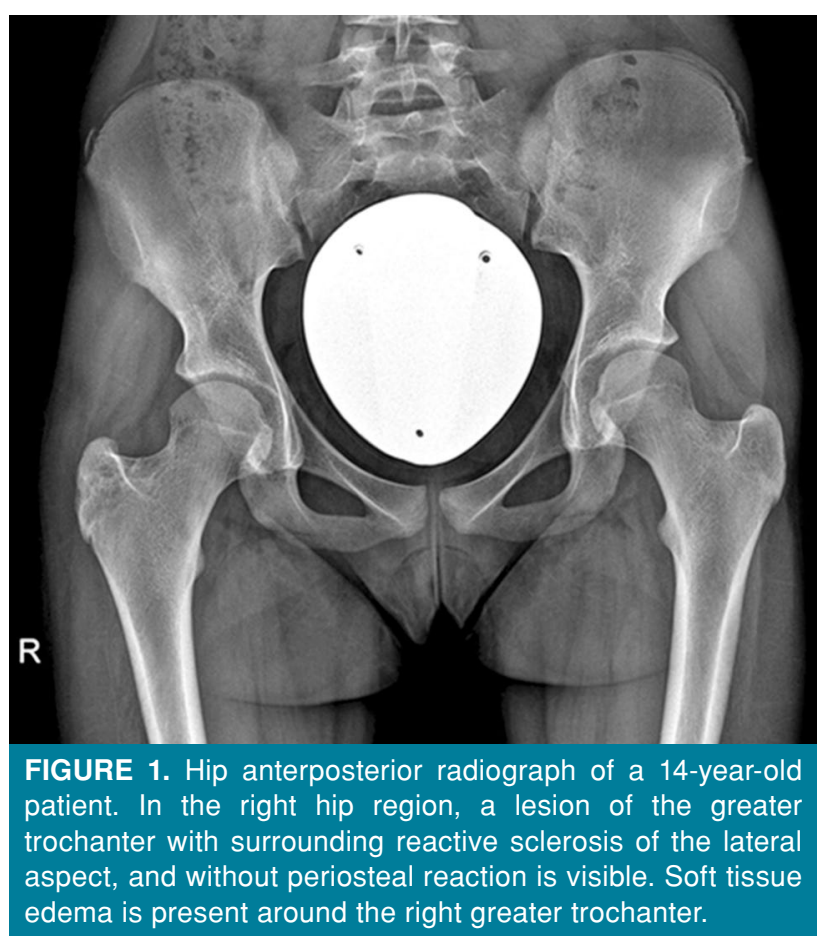



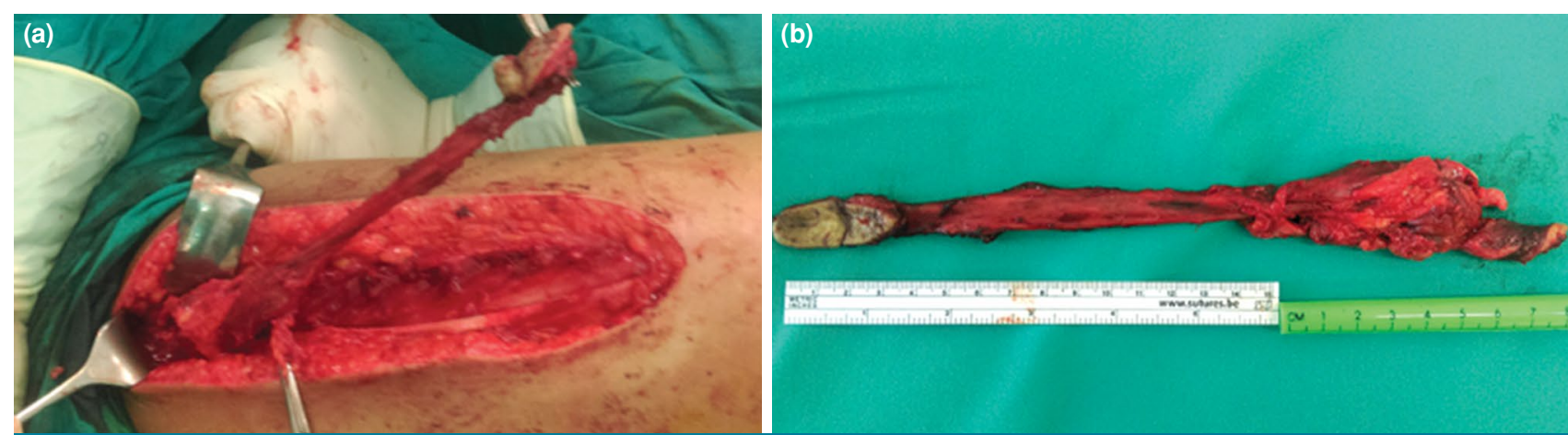

FIGURE 2. Intraoperative photographs. (a) Right thigh from lateral side - a long subfascial cutaneous fistula going from the middle third of the thigh to the greater trochanter region is visible; (b) Completely excised fistula and affected bursa before sending to pathology.

intraoperative finding of a cutaneous fistula, the skin incision was extended to track the fistula proximally. The fistula was marked with a probe, and a detailed exposure showed a subfascial fistula connected to the enlarged trochanteric bursa (Figure 2a). While operating on the trochanteric bursa, it partially ruptured and whitish liquid tissue, resembling pus was observed. At this point, TB was suspected and the material was taken for acid fast laboratory diagnostics. The fistula and trochanteric bursa were excised completely and sent to pathology (Figure $2 b$ ).

\section{Postoperative findings}

Postoperative intravenous antibiotic therapy was administered for 10 days with $2 \mathrm{~g}$ of ceftazidime. The direct microscopic smear examination from the specimen was negative for acid-fast bacilli. The pathology report described tissue changes characteristic for fistula, without specifying the underlying disease. The wound healed uneventfully. There was no longer any local tenderness and the patient was discharged home with recommendations to continue with antibiotic therapy.

Prior to first postoperative visit, the microbiology culture returned positive for Mycobacterium tuberculosis (M. tuberculosis). The experienced pathologist who reviewed the recent histological findings was notified. The specimen was re-reviewed and corrected identified M. tuberculosis and amended the report to possible TB infection (Figure 3). While discussing these results with the patient's mother, it was found that a close relative that lived with them was TB positive at the time of the index surgery eight years ago. The genotyping results of both $M$. tuberculosis strains were identical, confirming the likelihood of transmission. In the retrospective analysis of the initial cytology report from eight years ago, it was clearly reported as granulomatous inflammation with necrosis. Therefore, we concluded that the initial presentation with swelling and fluctuance on the right thigh was as a result of TTB. Hip and thigh magnetic resonance imaging (MRI) performed soon after surgery did not show any signs of TB.

\section{Pulmonology work-up and oral TB therapy}

Due to these findings, a complete pulmonology work-up was indicated. On pulmonary examination, the patient was immunocompetent, afebrile, had non-labored breathing, with normal breath sounds

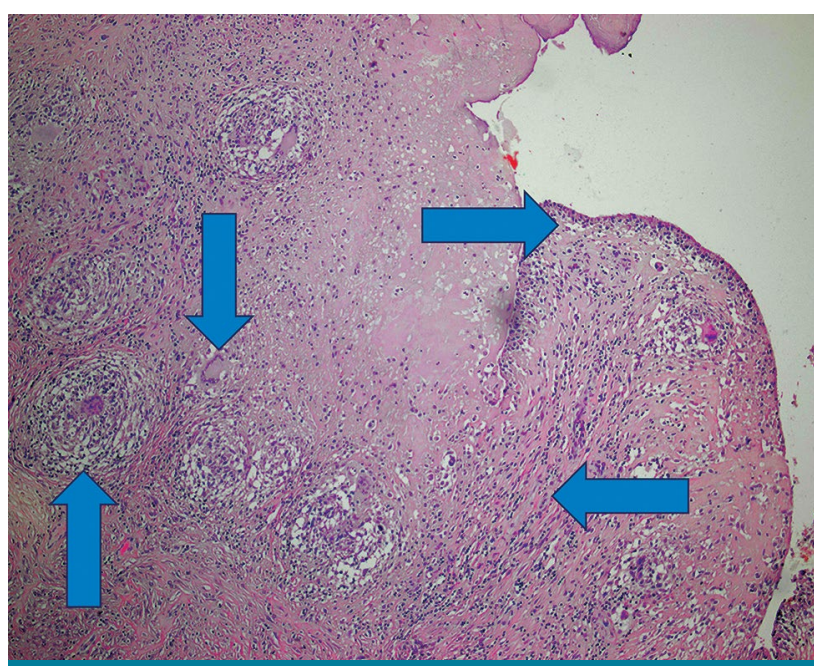

FIGURE 3. Microscopic analysis of trochanteric bursa biopsy showing ill-circumscribed granulomas composed of epithelioid and multinuclear cells and some mononuclear cells in the stroma. Some multinuclear cells are of Langhans type. Surface of the specimen is partly ulcerated with preserved synovial cells denoted by arrow. (H-E, $\times 100)$.

Down arrow-multinuclear giant cell of Langhans type, up arrow-poorly formed granuloma composed of epithelioid cells, left arrow-mononuclear inflammatory cells, right arrow-synovial cells on the surface. 
on lung auscultation. Bacille Calmette-Guérin (BCG) vaccination after birth was confirmed by her visible BCG scar. There was no history of previous pulmonary $\mathrm{TB}$, and her chest radiograph showed no signs of pathologic lesions. Culture of sputum samples were negative for $M$. tuberculosis. Oral TB treatment with four drugs was initiated (isoniazid $300 \mathrm{mg}$ daily; rifampicin $450 \mathrm{mg}$ daily; pyrazinamide $1 \mathrm{~g}$ daily; ethambutol $600 \mathrm{mg}$ daily) for two months and continued with two drugs (isoniazid $300 \mathrm{mg}$ daily; rifampicin $450 \mathrm{mg}$ daily) for 10 months.

The patient completed one year of TB therapy and was followed closely by a pulmonologist and orthopedic surgeon. Twenty months after surgery, she complained about snapping and pain of the right hip, which was successfully managed with physical therapy. At the most recent four-year follow-up, there was no evidence of local recurrence, and the patient returned to all normal activities of daily living.

An informed consent was obtained from the parents of the patient.

\section{DISCUSSION}

The presented case of TTB took eight years to be correctly diagnosed and successfully treated. Unfortunately, during this delay, a great burden to the patient and family was created. Therefore, we believe that this report highlights all diagnostic pitfalls present in this case and attempts to explain why TTB remained neglected.

Based on the data published in the last Global Tuberculosis Report by the World Health Organization (WHO), the number of new TB cases has trended down over the last decades. Nevertheless, in developing countries, TB remains an important source of mortality. ${ }^{[2]}$ The ongoing migrant crisis may also affect the future TB incidence in Europe. In Croatia, the latest report on TB states the incidence of TB to be 9.1 per 100,000 population, while musculoskeletal TB was reported in only one patient in 2018. ${ }^{[9]}$

Presentation of musculoskeletal TB may be insidious over a long period, as the diagnosis remains elusive and delayed. Vertebral involvement (i.e., tuberculous spondylitis, or Pott's disease) is the most common type of skeletal $\mathrm{TB}$, accounting for about half of all cases of musculoskeletal TB. ${ }^{[1]}$ However, when other sites are affected, such as the trochanteric bursa, TB is not initially considered in the differential diagnosis.
A standardized search of the published data on publicly available search platforms was performed to identify studies dealing with TTB. To the best of our knowledge, only a few cases of TTB in children were reported (Table I). ${ }^{[5,7,8,10]}$ The first description of, what is now considered to be TTB in a child, was given by Macnab, ${ }^{[10]}$ a surgeon at Suffolk General Hospital, United Kingdom, in 1870. In his report, following minor trauma, the child's hip was slightly swollen over the greater trochanter, hot, sore on palpation, while walking was painful. Recommended therapy was rest, supportive ambulation, and immobilization. However, six months later, swelling over the hip had suddenly become painful, rapidly increased in size, and erupted, discharging a large quantity of pus. Macnab, then, instructed carbolic acid to be injected into the abscess three times daily, and series of dressing to be applied to the wound. Finally, after one month, the wound over the hip diminished in size, healthy granulation tissue was present, with little discharge. Hip range of motion was symmetric to the unaffected side. According to the physical exam and treatment described, one could notice that Macnab, indeed, witnessed the natural course of TTB. There is a remarkable resemblance between our recent case and Macnab's description 150 years ago what should now provoke disappointment to all physicians involved.

In the first half of the $20^{\text {th }}$ century, TTB was not uncommon. ${ }^{[5,6]}$ Among 15 cases of TTB, McMurray ${ }^{[5]}$ reported two of them in children. Similar to our case, a 14-year-old girl complained to a thigh swelling and pain for more than a year. Affected bursa was aspirated, but still the patient developed bony erosion of the greater trochanter in a one-year follow-up. Therefore, the author strongly emphasizes that greater trochanteric bone lesions were often found secondary to TB infection of the trochanteric bursa. Seen backwards, the described erosions of the greater trochanter on radiographs in our patient should raise suspicion of TTB even before surgical intervention. Nevertheless, the diagnostic challenge in regards to TB of the bone, joint, and bursa in children was recognized long ago. In 1931, Milgram ${ }^{[4]}$ reported the diagnostic inaccuracy between LeggCalvé-Perthes (LCP) disease and TB of the hip. However, currently, LCP disease is not a diagnostic challenge with TB due to imaging methods used. In the last three decades, two radiological studies reported on two adolescent patients with TTB. ${ }^{[7,8]}$ As in our case, X-ray findings indicate the slight lytic changes of the greater trochanter and soft tissue reaction. ${ }^{[7,8]}$ 


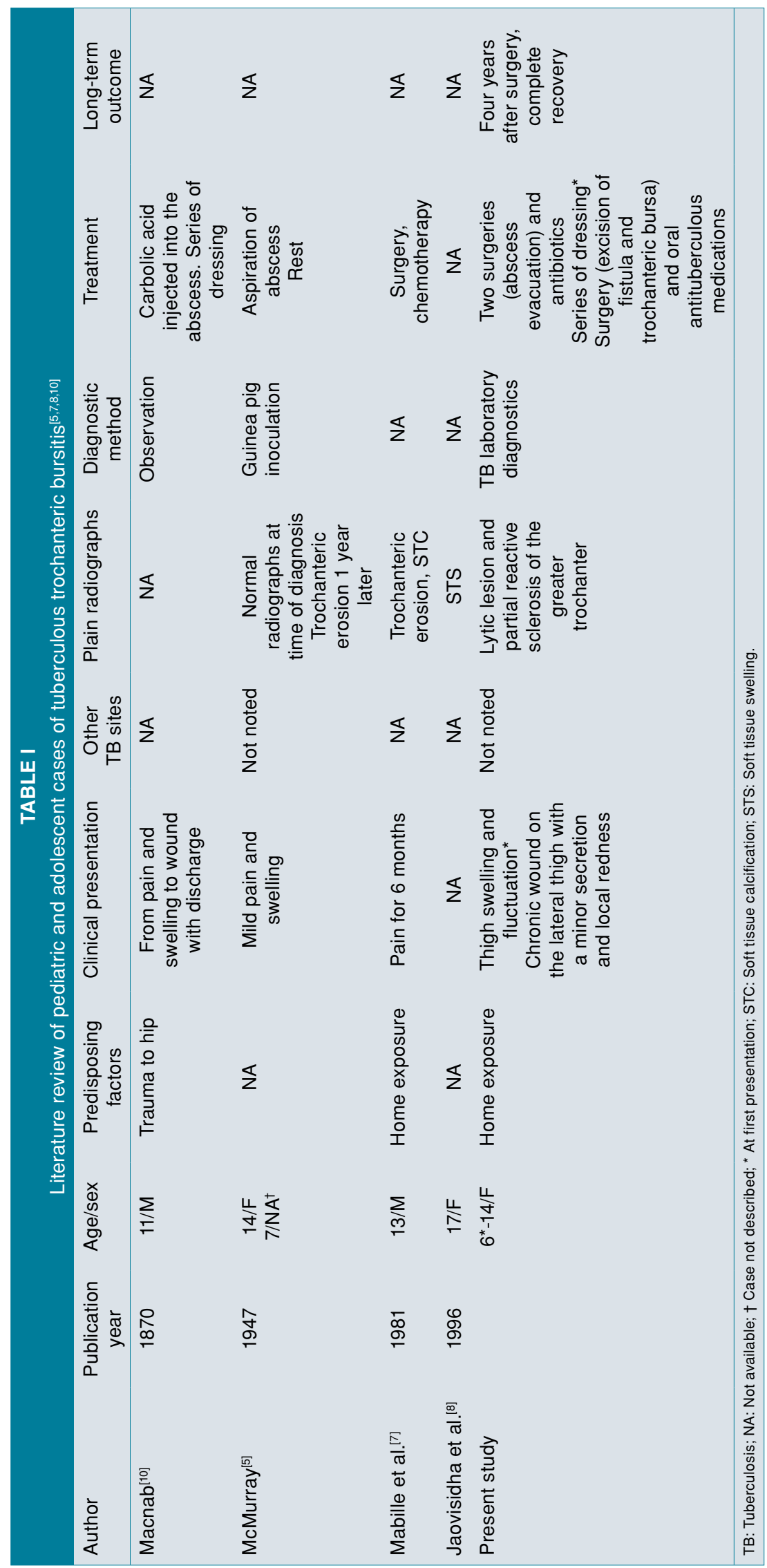


As seen in Table I, in the present time, the lack of familiarity with TTB in children comes from its' extremely low incidence and, therefore, data paucity in orthopedic literature. ${ }^{[3,5,7,8,10]}$

Tuberculous trochanteric bursitis can occur through hematogenous transmission from a remote site of infection or by direct spread from an adjacent infectious site. According to published data, the primary focus of TTB could be identified in nearly half of the cases. ${ }^{[3]}$ In our case, chest radiographs showed no abnormalities, and there were no MRI findings suggesting nearby structures as infectious foci. Moreover, no other present or past evidence of any other foci of TB were found. Thus, we assumed that hematogenous spread of $M$. tuberculosis bacilli after inhalation; however, without pulmonary involvement, it led to the development of primary TTB.

When analyzing our case, several clinical missteps were identified, starting with the initial presentation. At first, when a six-year-old child presents with swelling and fluctuance over the thigh, without history of trauma, the differential should include infection and tumor. Therefore, it is not surprising that the diagnosis of musculoskeletal $\mathrm{TB}$ is often mistaken as malignancy. ${ }^{[1]}$ However, chronic wounds with secretion and minor pain should always raise suspicion on musculoskeletal TB. ${ }^{[1,5]}$ Despite that, the treating orthopedic surgeon overlooked the lack of improvement on antibiotic therapy, misread pathology report, and approached this case as a recurrent pyomyositis.

In this case, the crucial event leading to proper diagnosis of TTB was, when the whitish liquid, resembling pus was noted intraoperatively, and TB smear was taken. A definitive diagnosis of TTB requires the obtaining of specimens from the trochanteric bursa for TB microscopy and culture, while the finding of granulomas and cultivation of M. tuberculosis are the gold standards for diagnosing TTB. ${ }^{[11]}$

Optimal treatment for TTB remains debatable and involves mainly TB drugs, which are administered for 6 to 18 months, combined with surgical treatment. ${ }^{[12]}$ Although TB drugs can cure the disease at any stage, it is worth noting that, in some patients, the treatment of TTB with TB drugs alone may lead to TB recurrence. ${ }^{[13]}$

In conclusion, we emphasize that $\mathrm{TB}$ still represents a diagnostic and treatment challenge, particularly in areas of low incidence. Therefore, it is a responsibility of orthopedic surgeons to consider
TB as a possible source of disturbances in children, to avoid cases of neglected TTB in the future.

\section{Acknowledgement}

We would like thank to Asst. Prof. Martin J. Morrison, III, MD; (Educational Consultant for DePuy Synthes Spine and Educational Consultant for OrthoPediatrics); Loma Linda University Department of Orthopedic Surgery Loma Linda, CA 92354 USA for editing and writing assistance.

\section{Declaration of conflicting interests}

The authors declared no conflicts of interest with respect to the authorship and/or publication of this article.

\section{Funding}

The authors received no financial support for the research and/or authorship of this article.

\section{REFERENCES}

1. Leonard MK Jr, Blumberg HM. Musculoskeletal tuberculosis. In: Schlossberg D, editor. Tuberculosis and nontuberculous mycobacterial infections. 7th ed. Washington: American Society for Microbiology; 2017. p. 371-92.

2. TB diagnosis and treatment. In: Global tuberculosis report 2019. Chapter 4. Geneva: World Health Organization; 2019. p. 73-109.

3. Crespo M, Pigrau C, Flores X, Almirante B, Falco V, Vidal $\mathrm{R}$, et al. Tuberculous trochanteric bursitis: report of 5 cases and literature review. Scand J Infect Dis 2004;36:552-8.

4. Milgram JE. Diagnostic inaccuracy in tuberculosis of bone, joint and bursa. Jama 1931;97:232-5.

5. McMurray B. Tuberculosis of the great trochanter. Br Med J 1947;2:492-4.

6. Jefferson HC, Palmer JN, Phillips CW. Tuberculosis of the greater trochanter and its bursa treatment with streptomycin and surgery. J Int Coll Surg 1950;13:361-6.

7. Mabille JP, Collombier B, Magnet JL, Strauss J. Tuberculous trochanteritis: Radiological diagnosis (author's transl). J Radiol 1981;62:25-30.

8. Jaovisidha $S$, Chen C, Ryu KN, Siriwongpairat P, Pekanan $\mathrm{P}$, Sartoris DJ, et al. Tuberculous tenosynovitis and bursitis: imaging findings in 21 cases. Radiology 1996;201:507-13.

9. Bakteriološka diagnostics of tuberculosis 2018. godini Bacteriological Diagnostics of Tuberculosis in 2018. In: Croatian health statistics yearbook 2018. Zagreb, Croatian: Institute of Public Health; 2019. p. 213-5.

10. Macnab R. On the simulation of hip-joint disease by suppuration of the bursa over the trochanter major. The Lancet 1870;96:666-7.

11. McNerney R, Cunningham J, Hepple P, Zumla A. New tuberculosis diagnostics and rollout. Int $\mathrm{J}$ Infect Dis 2015;32:81-6.

12. Ramos-Pascua LR, Carro-Fernández JA, Santos-Sánchez JA, Casas Ramos P, Díez-Romero LJ, Izquierdo-García FM. Bursectomy, curettage, and chemotherapy in tuberculous trochanteric bursitis. Clin Orthop Surg 2016;8:106-9.

13. Soro Marín S, Sánchez Trenado MA, Mínguez Sánchez MD, Paulino Huertas M, García Morales PV, Salas Manzanedo V. Trochanteric bursitis due to tuberculosis in an immunocompetent young woman. Reumatol Clin 2012;8:34-5. 